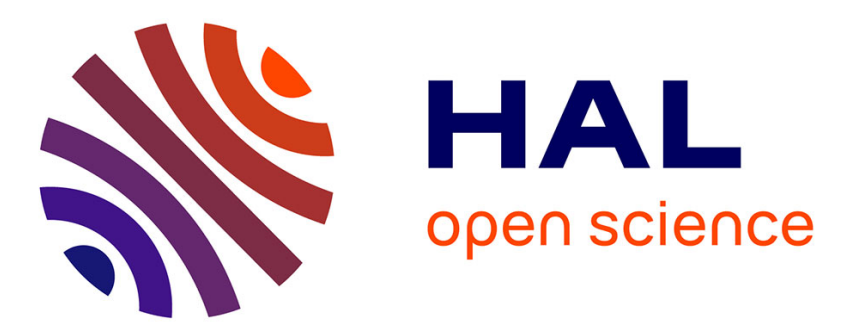

\title{
Intrinsic damping of spin waves by spin current in conducting two-dimensional systems
}

\author{
J. Gomez, Florent Pérez, E. M. Hankiewicz, Bernard Jusserand, G. \\ Karczewski, T. Wojtowicz
}

\section{- To cite this version:}

J. Gomez, Florent Pérez, E. M. Hankiewicz, Bernard Jusserand, G. Karczewski, et al.. Intrinsic damping of spin waves by spin current in conducting two-dimensional systems. Physical Review B: Condensed Matter and Materials Physics (1998-2015), 2010, 81 (10), pp.100403(R). 10.1103/PhysRevB.81.100403 . hal-01229219

\section{HAL Id: hal-01229219 https://hal.science/hal-01229219}

Submitted on 1 Jun 2016

HAL is a multi-disciplinary open access archive for the deposit and dissemination of scientific research documents, whether they are published or not. The documents may come from teaching and research institutions in France or abroad, or from public or private research centers.
L'archive ouverte pluridisciplinaire HAL, est destinée au dépôt et à la diffusion de documents scientifiques de niveau recherche, publiés ou non, émanant des établissements d'enseignement et de recherche français ou étrangers, des laboratoires publics ou privés. 


\title{
Intrinsic damping of spin waves by spin-current in conducting two-dimensional systems
}

J. Gómez ${ }^{1}$, F. Perez ${ }^{1}$, E. M. Hankiewicz², B. Jusserand ${ }^{1}$, G. Karczewski ${ }^{3}$, T. Wojtowicz ${ }^{3}$

${ }^{1}$ Institut des Nanosciences de Paris, CNRS/Université Paris VI, Paris, France.

${ }^{2}$ Institut für Theoretische Physik und Astrophysik, Universität Würzburg, Germany and

${ }^{3}$ Institute of Physics, Polish Academy of Sciences, Warsaw, Poland.

(Date textdate)

\begin{abstract}
Angle resolved magneto-Raman scattering has been performed on spin-polarized two dimensional electron gas embedded in $\mathrm{Cd}_{1-x} \mathrm{Mn}_{x}$ Te quantum wells to explore the intrinsic damping of propagating spin waves modes with in plane momentum $q$. The damping rate $\eta$ follows a quadratic law $\eta=\eta_{0}+\eta_{2} q^{2}$ due to losses in the spin-current driven by the magnetization in qualitative agreement with Phys.Rev.B 78, 020404(R) 2009. As a consequence, the propagation length of a spin wave in a conducting system has an intrinsic maximum of importance for spin-wave based spintronics.
\end{abstract}


Avoiding dissipation of spin currents in the context of spin-based electronics, has become an important challenge today. Indeed, in devices using spin-polarized currents ${ }^{1}$, the spin of carriers is displaced together with their charge. This results in Joule dissipation. Pure spin currents (PSC), without net charge current have been recently proposed in non-magnetic materials with spin-orbit interaction ${ }^{2}$. However, they suffer from an intrinsic damping, the so-called spin Coulomb drag (SCD) ${ }^{34}$, which results from the friction between carriers with anti-parallel spins. The SCD is very efficient in semiconductors where PSCs involve longitudinal (with respect to the spin-quantization axis) spin degrees of freedom. An alternative would be to make use of transverse spin degrees of freedom, naturally good candidate for pure spin information transport. For example, spin waves in ferromagnets, may be the means to transmit and modify a logical information ${ }^{5}$. They are also the elemental components of transverse PSCs. In this frame, understanding the intrinsic limitations of spin waves propagation is crucial. Recently, the attention to this topic has been drawn in Ref. ${ }^{6}$, where it was found that a spin wave of momentum $q$ in a spin-polarized conducting system had an intrinsic damping proportional to $q^{2}$.

We have performed angle resolved electronic resonant Raman scattering (ERRS) experiments on a two-dimensional conducting spin-polarized system to evidence, for the first time, the $q^{2}$ intrinsic damping of propagating spin waves modes. We also completed the theory of Ref. ${ }^{6}$ by exact dynamics considerations. The damping rate we found experimentally varies as $q^{2}$ as predicted in Ref. ${ }^{6}$, but additional corrections were necessary to match quantitatively the data without fitting parameters.

We studied high mobility two dimensional electron gases (2DEG) embedded in $\mathrm{Cd}_{1-x} \mathrm{Mn}_{x} \mathrm{Te} / \mathrm{Cd}_{0.8} \mathrm{Mg}_{0.2} \mathrm{Te}$ quantum wells ${ }^{7}$. Such systems have been recently introduced as a test-bed system for spin-excitations of the spin polarized 2DEG (SP2DEG) ${ }^{89}$. Indeed, the s-d exchange coupling of conduction electron with localized electrons of the Mn atoms provide a giant Zeeman energy ${ }^{10}$ to the $2 \mathrm{DEG}$ :

$$
Z(B, T)=\bar{x} N_{0} \alpha_{e}\left\langle S_{z}(B, T)\right\rangle-\left|g_{e}\right| \mu_{B} B
$$

where $N_{0}$ is the cation sites density, $\alpha_{e}$ is the exchange coupling between the conduction electron of the well and the localized electrons on Mn impurities $\left(N_{0} \alpha_{e}=-0.22 \mathrm{eV}\right)^{10}$. $\left\langle S_{z}(B, T)\right\rangle$ is the thermal average spin of a single $\mathrm{Mn}$ atom given by the modified Brillouin function, $\bar{x}$ is the effective Mn concentration (for low $x, \bar{x} \simeq x$ ), $g_{e}$ is the normal electron 
$g$-factor and $\mu_{B}$ is the Bohr magneton $\left(\mu_{B}>0\right)$. In order to keep alloy disorder low and high electron mobility $\left(\mu^{\sim} 10^{5} \mathrm{~cm}^{2} \mathrm{Vs}\right), x$ remained below $1 \%$ and the electron sheet density $n_{2 D}$ ranged in between 1.5 and $4 \times 10^{11} \mathrm{~cm}^{-2}$. Depending on the Mn nominal concentration $x N_{0}$ and $n_{2 D}$, the maximum spin polarization degree $\zeta=\left(n_{\uparrow}-n_{\downarrow}\right) / n_{2 D}$ can reach $80 \%$ for a magnetic field $B$ below $4 \mathrm{~T}$, such that, when $B$ is applied parallel to the quantum well plane, the Landau orbital quantization is kept negligible. Therefore, such SP2DEG are "artificial" conducting paramagnet having the spin-polarization degree of a conducting ferromagnet. Morover, semiconductor quantum wells exhibit well defined optical resonances which allow ERRS measurements to be performed ${ }^{11}$. ERRS is a powerful tool to access wavevector resolution of the spin excitations spectrum ${ }^{8}$. Over the last decades, general knowledge on electron gases low-energy excitations has been considerably improved by ERRS study of high mobility unpolarized $2 \mathrm{DEG}^{12}$. We claim that, the SP2DEG system investigated here is able to provide general knowledge on spin waves in spin-polarized conducting systems, in particular to the subject here : the $q^{2}$ damping.

We start with the theoretical description of the intrinsic damping and consider the Hamiltonian of the above spin polarized 2DEG :

$$
\hat{H}_{S P 2 D E G}=\hat{H}_{\text {Kin }}+\hat{H}_{\text {Zeeman }}+\hat{H}_{\text {Coulomb }}
$$

where $\hat{H}_{K i n}+\hat{H}_{Z e e m a n}=\sum_{\mathbf{k}, \sigma}\left(E_{\mathbf{k}}+\sigma Z\right) c_{\mathbf{k}, \sigma}^{+} c_{\mathbf{k}, \sigma}, E_{\mathbf{k}}=\frac{\hbar^{2} k^{2}}{2 m^{*}}$ is the kinetic energy of the single electron state $|\mathbf{k} \sigma\rangle$ with in plane wavevector $\mathbf{k}$ and spin $\sigma= \pm \frac{1}{2}$. Z is the Zeeman energy given by Eq.(1). $c_{\mathbf{k} \sigma}^{(+)}$are creation-anihilation operators. Spin waves are transverse precession modes of the magnetization described by the operators $\hat{S}_{+, \mathbf{q}}=\iint \hat{\mathbf{S}}_{+}(\mathbf{r}) e^{-i \mathbf{q} \cdot \mathbf{r}} d^{2} r$, space Fourier-transform of the transverse spin-density $\hat{\mathbf{S}}_{+}(\mathbf{r})$. As $\hat{S}_{+, \mathbf{q}}=\sum_{\mathbf{k}} c_{\mathbf{k}-\mathbf{q}, \uparrow}^{+} c_{\mathbf{k}, \downarrow}, \mathrm{a}$ magnetization can also be seen as a coherent superposition of individual spin-flip electronhole pairs : $c_{\mathbf{k}-\mathbf{q}, \uparrow}^{+} c_{\mathbf{k}, \downarrow}|0\rangle$. In presence, of a perturbing rotating magnetic field $b_{+}(\mathbf{r}, t)=$ $b_{+\mathbf{q} \omega} e^{i \mathbf{q} \cdot \mathbf{r}-i \omega t}$, the equation of motion for the magnetization $\mathfrak{m}_{+\mathbf{q} \omega}=\left\langle\hat{S}_{+, \mathbf{q}}\right\rangle_{\omega}$ with $\omega$ and $\mathbf{q}$ Fourier components writes exactly:

$$
\omega \mathfrak{m}_{+\mathbf{q} \omega}=\omega_{0} \mathfrak{m}_{+\mathbf{q} \omega}+\mathbf{q} \cdot\left\langle\hat{\jmath}_{+, \mathbf{q}}\right\rangle_{\omega}+M_{0} g_{e} \mu_{B} b_{+\mathbf{q} \omega}
$$

In Eq.(3), $Z=\hbar \omega_{0}$ and $M_{0}=\left\langle\hat{S}_{z, \mathbf{q}=\mathbf{0}}\right\rangle_{0}$ is the equilibrium 2DEG magnetization. The first term in the rhs of Eq.(3) arises from $\left[\hat{S}_{+, \mathbf{q}}, \hat{H}_{\text {Zeeman }}+\hat{H}_{\text {Coulomb }}\right]$. The second 
term in the rhs of Eq.(3) is the discussed effect and is the commutator $\left[\hat{S}_{+, \mathbf{q}}, \hat{H}_{\text {Kin }}\right]=$ $\sum_{\mathbf{k}}\left(E_{\mathbf{k}}-E_{\mathbf{k}-\mathbf{q}}\right) c_{\mathbf{k}-\mathbf{q}, \uparrow}^{+} c_{\mathbf{k}, \downarrow}$, written in terms of $\hat{\jmath}_{+, \mathbf{q}}=\frac{\hbar}{m^{*}} \sum_{\mathbf{k}}\left(\mathbf{k}-\frac{\mathbf{q}}{2}\right) c_{\mathbf{k}-\mathbf{q}, \uparrow}^{+} c_{\mathbf{k}, \downarrow}$, the Fourier transform of the transverse spin-current density. Hence, if $\mathbf{q} \neq 0$, the kinetic Hamiltonian couples the magnetization to the spin current. One is left with exploring the dynamics of the spin-current. Despite its collective character, this quantity is not conserved by Coulomb, neither it is by the kinetic part because of the spread of velocities of individual pairs. This makes its dynamics governed by individual dynamics of $c_{\mathbf{k}-\mathbf{q}, \uparrow}^{+} c_{\mathbf{k}, \downarrow}$. The latter experiences scattering due to disorder, but also scattering due to Coulomb which couples the single electron-hole pair $c_{\mathbf{k}-\mathbf{q}, \uparrow}^{+} c_{\mathbf{k}, \downarrow}$ with multiple electron-hole pairs having the same total spin $(+1)$. The spin Coulomb drag theory ${ }^{3}$ describes to leading order the efficiency of multi-pair scattering. Amongst scatterings, indivividual pairs are sensitive to a local magnetic field, addition of the external one and the Coulomb exchange-correlation field due to other individual pairs. The latter field brings the new theoretical contribution of this work, which was not taken into account in Ref. ${ }^{6}$. The spin-current equation of motion writes then (to first order in $\left.b_{+\mathbf{q} \omega}\right)$ :

$$
\left\langle\hat{\jmath}_{+, \mathbf{q}}\right\rangle_{\omega}=-\mathbf{q} \tilde{\sigma}_{\perp}\left(\frac{1}{2} g_{e} \mu_{B} b_{+\mathbf{q} \omega}-U \mathfrak{m}_{+\mathbf{q} \omega}\right)
$$

where $\tilde{\sigma}_{\perp}=-\frac{\left\langle\left\langle\hat{\jmath}_{+, \mathbf{q}} ; \hat{\jmath}_{-,-\mathbf{q}}\right\rangle\right\rangle_{\omega}}{\omega-\omega_{0}}$ is the spin conductivity linking $\left\langle\hat{\jmath}_{+, \mathbf{q}}\right\rangle_{\omega}$ to the gradient of the magnetic field ${ }^{6}$, which is enhanced by the local exchange-correlation field $-U \mathfrak{m}_{+\mathbf{q} \omega}$. $U$ is the transverse local field factor. In the local spin-density approximation, $U=-\frac{2}{M_{0}} \frac{\partial E_{x c}}{\partial M_{0}}$, where $E_{x c}$ is the exchange-correlation part of the ground state energy ${ }^{9}$. This local field factor is also responsible for the Zeeman energy enhancement ${ }^{13}: Z^{*}=\hbar \omega_{0}^{*}=Z-U M_{0}$. Hence, it is clear from $\operatorname{Eqs}(3)$ and (4) that any loss in the spin-current (imaginary contribution in $\tilde{\sigma}_{\perp}$ ) introduces a damping of the magnetization precession, proportional to $q^{2}$. The resulting Gilbert damping $\alpha$ is given by :

$$
\begin{gathered}
\alpha=\frac{\hbar \omega_{0}^{2}}{2 M_{0}} \lim _{\omega \rightarrow 0} \frac{\operatorname{Im} \chi_{+}}{\omega} \\
=-\frac{q^{2} \hbar}{4 M_{0}}\left[\sigma_{\perp}+\sigma_{\perp}^{\prime} \omega_{0}-\frac{2 M_{0}}{\hbar} U\left(\sigma_{\perp}^{\prime}+2 \frac{\sigma_{\perp}}{\omega_{0}}\right)\right] \\
=\frac{q^{2} \hbar}{2 m^{*}|\zeta|} \frac{\omega_{0}^{*}}{\omega_{0}} \frac{3 \tau}{\left(\omega_{0}^{*} \tau\right)^{2}+1}\left[\frac{\omega_{0}^{*}}{\omega_{0}}-\frac{\left(\omega_{0}^{*} \tau\right)^{2}+\frac{1}{3}}{\left(\omega_{0}^{*} \tau\right)^{2}+1}\right]
\end{gathered}
$$


where $\chi_{+}=\frac{\mathfrak{m}_{\mathbf{q} \mathbf{\omega} \omega}}{g_{e} \mu_{B} b_{+\mathbf{q} \omega}}$ is the spin-susceptibility, $\sigma_{\perp}=\operatorname{Im} \tilde{\sigma}_{\perp}(0)$ and $\sigma_{\perp}^{\prime}=\lim _{\omega \rightarrow 0} \frac{\operatorname{Im} \tilde{\sigma}_{\perp}(\omega)}{\omega}$. Compared to Ref. ${ }^{6}$, the second, third and fourth terms in Eq.(6) are the new contributions introduced here. Obviously the last two are due to the Coulomb local field, while the second term is due to dynamical properties of the spin conductivity. Further considerations on $\tilde{\sigma}_{\perp}$ show that disorder and Coulomb scattering give additive imaginary contributions in the spin current - spin current reponse ${ }^{6}\left\langle\left\langle\hat{\jmath}_{+, \mathbf{q}} ; \hat{\jmath}_{-,-\mathbf{q}}\right\rangle\right\rangle_{\omega}$. Therefore, the scattering time $\tau$ introduced in Eq.(7) is given by $\frac{1}{\tau}=\frac{1}{\tau_{d i s}}+\frac{1}{\tau_{e e}}, \frac{1}{\tau_{d i s}}$ and $\frac{1}{\tau_{e e}}$ are respectivley, the disorder and transverse-spin Coulomb drag scattering rates.

Evidence of this universal $q^{2}$ behavior has been performed in the high mobility SP2DEG described above. Since, the well defined spin waves modes has been successfully observed in these quantum wells ${ }^{8}$, this material is a perfect candidate to investigate the damping. The sketch on Fig.1 depicts the experimental geometry : the external magnetic field (B) is applied in the $z$ direction parallel to the quantum well plane and the average angle $\theta$ of the incoming and back-scattered light wavevectors with respect to the normal direction can be tuned to make the in plane Raman transferred wavevector $q=\frac{4 \pi}{\lambda} \cos \frac{\beta}{2} \sin \theta$ vary in the range $0<q<16 \mu \mathrm{m}^{-1}, \beta \simeq 5^{\circ}$ and $\lambda$ is the incoming light wavelength.

When the polarizations of the incoming and scattered photons are crossed, the Raman spectrum is determined by the transverse spin-susceptibility $\operatorname{spectrum} \operatorname{Im} \chi_{+}(q, \omega)$. This is always true when out of resonance. In resonance, however, it remains valid if $q$ is small compared to wavevectors of electrons involved in optical processes (typically greater than the Fermi wavevector $k_{F}$ ) and when the intermediate state lifetime is shorter than the one of the excitation considered. The former condition is immediatley fulfilled as $q$ remains much smaller than typical $k_{F}^{\sim} 150 \mu \mathrm{m}^{-1}$.

In Fig.1(a), we have plotted typical crossed polarized Raman spectra obtained for increasing $q$ and fixed external magnetic field at superfluid He bath temperature $(T \sim 2 . \mathrm{K})$. These spectra present a clear dispersive Raman line associated to the spin flip wave (SFW) excitation of the SP2DEG ${ }^{13}$. In Fig.1(b), we show the resonant behavior of the Raman peak when tuning the laser wavelength across the optical resonance. This shows how the resonance width is 20 times larger than the SFW line in the Raman spectra. Hence, we can consider that Raman spectra give access to $\operatorname{Im} \chi_{+}(q, \omega)$ and extract from these data both 
q2/VersionFinale/FIG1.wmf

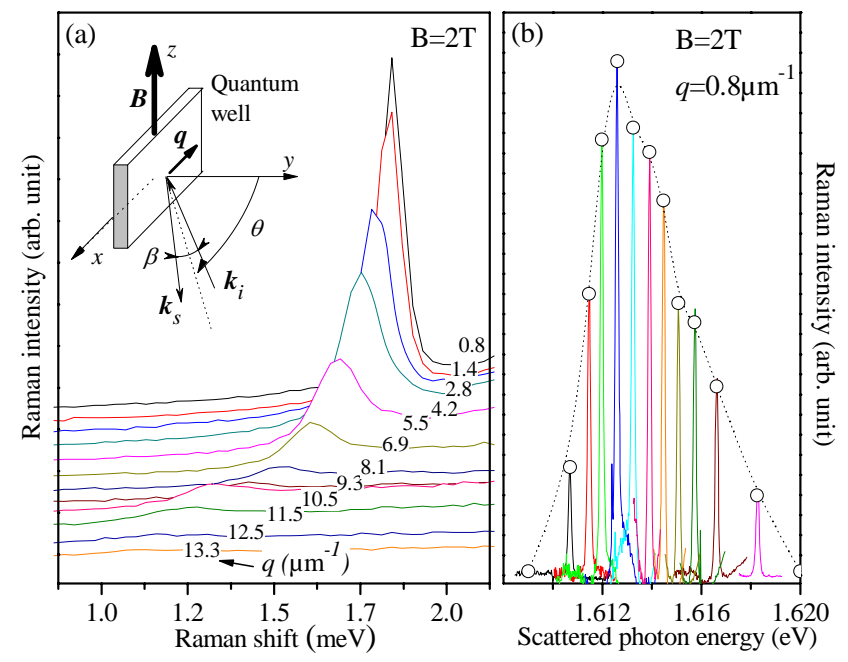

FIG. 1: (color online) (a) Typical crossed polarized Raman spectra obtained on sample A at $B=2 \mathrm{~T}$ and for different values of $q$. The single Raman line is the SFW. A sketch of the scattering geometry shows the angles definition. Incoming photon is polarized parallel to $\mathbf{B}(\pi)$, while the scattered one is polarized perpendicular to $\mathbf{B}(\sigma)$. (b) Spectra obtained by shifting the Laser wavelength. Amplitude variations of the Raman line reveal the optical resonance width.

the SFW energy $\left(\hbar \omega_{S F W}\right)$ and the line width $\eta q$-dependance. As shown in Fig.2(a) the former is well reproduced by the formula ${ }^{9}$ below :

$$
\hbar \omega_{S F W}=Z-\frac{1}{|\zeta|} \frac{Z}{Z^{*}-Z} \frac{\hbar^{2}}{2 m^{*}} q^{2}
$$

when $m^{*}=0.105 m_{e}$ is the CdTe conduction electron effective mass. This provides another confirmation for the identification of the Raman line. Following previous assessments, we have extracted the linewidth of the Raman line after background subtraction and deconvolution with the spectrometer response (Voigt profile of apparent FWHM $62 \mu \mathrm{eV}$ ) to get the damping rate of magnetization modes. The deconvoluted linewidth $\eta$ of the Raman line is plotted in Fig.2(b) as a function of $q^{2}$ for the same conditions as the dispersions plotted in Fig.2(a). It shows that, in the explored range of wave vectors $\left(q \ll k_{F}\right)$, the linewidth $q$-dependence is very well reproduced by a parabolic function :

$$
\eta=\eta_{0}+\eta_{2} q^{2}
$$

In Eq. $(9), \eta_{0}$ gives the homogenous mode $(q=0)$ damping and $\eta_{2}$ is inferred to be linked to the $q^{2}$ damping of Eq.(5) : $\eta_{2}=2 \hbar \omega_{0} \alpha / q^{2}$. Indeed, $\eta_{2}$ has obviously a strong magnetic 
q2/VersionFinale/FIG2.wmf

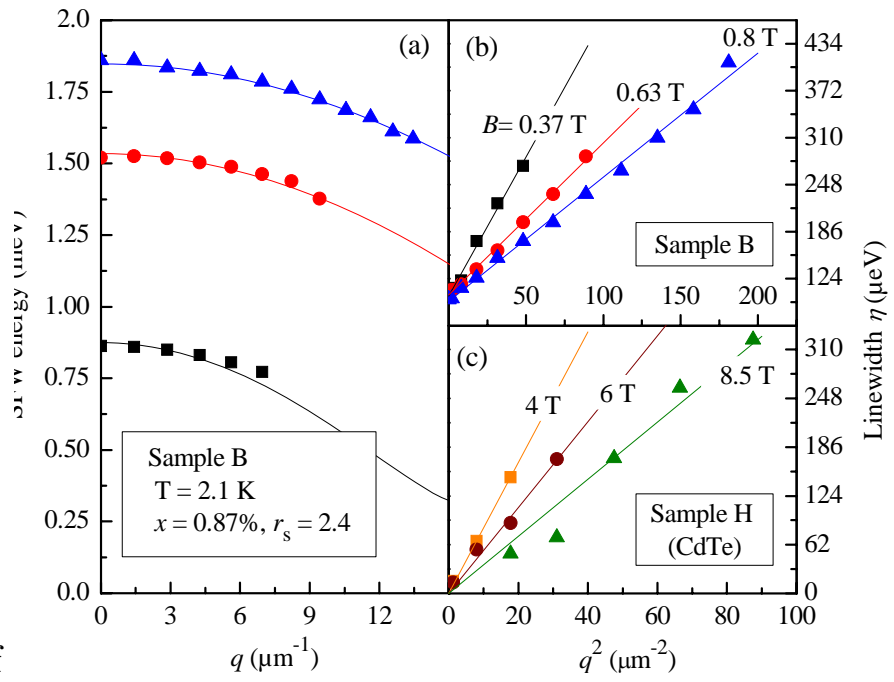

FIG. 2: (color online) Typical SFW energy (a) and linewidth (b) $q$-dependence obtained on sample B $\left(x=0.87 \%, r_{s}=2.4\right)$ for $B=0.37,0.63$ and 0.8 T. In agreement with Eqs.(8) and (9) the data follow a parabolic behavior.(c) Linewidth $q$-dependence obtained on CdTe sample $\mathrm{H}$

field dependence. We may ask if the presence of Mn impurities can be the cause of $\eta_{2}$ ? It is already known that Mn spin fluctuations damp the homogeneous mode ${ }^{14}$. But, in our samples, the typical Mn average distance $\bar{d} \sim 0.4 \mathrm{~nm}$ is far smaller than the minimum magnetization wavelength probed in the Raman experiment $(q \bar{d} \ll 1)$. Hence, Mn damping is expected to be constant in the explored range of $q$ and not present in $\eta_{2}$. We confirmed by carrying the same measurements on a CdTe quantum well (without Mn). Fig.2(c) shows that the same $q^{2}$ law has been found and that $\eta_{2}$ has the same order of magnitude. Without $\mathrm{Mn}$, the Zeeman energy is much lower and requires higher fields, this reduces the range of explored spin-polarization. Consequently, we claim that the Mn damping is, here, present in $\eta_{0}$ and that $\eta_{2}$ originates from the general behavior described above. Let us compare the experimental $\eta_{2}$ with the theoretical one.

Fig. 3 shows the behavior of the $q^{2}$-damping $\eta_{2}$ with the polarization or equivalently with the Zeeman pulsation $\omega_{0} \cdot \eta_{2}$ determined on all the studied samples is compared with the calculations of Eqs.(7)-(9) and Ref. ${ }^{6}$. The accuracy of the measurments is not able to separate variations of $\eta_{2}$ with the electron density within the small range explored here $\left(r_{s}=5 \mathrm{~nm} / \sqrt{\pi n_{2 D}} \in[1.7,2.4]\right)$. But within the experimental error, all the CdMnTe data follows the same power-law even if the Mn concentration varies like 1:5. This confirms 
q2/VersionFinale/FIG3.wmf

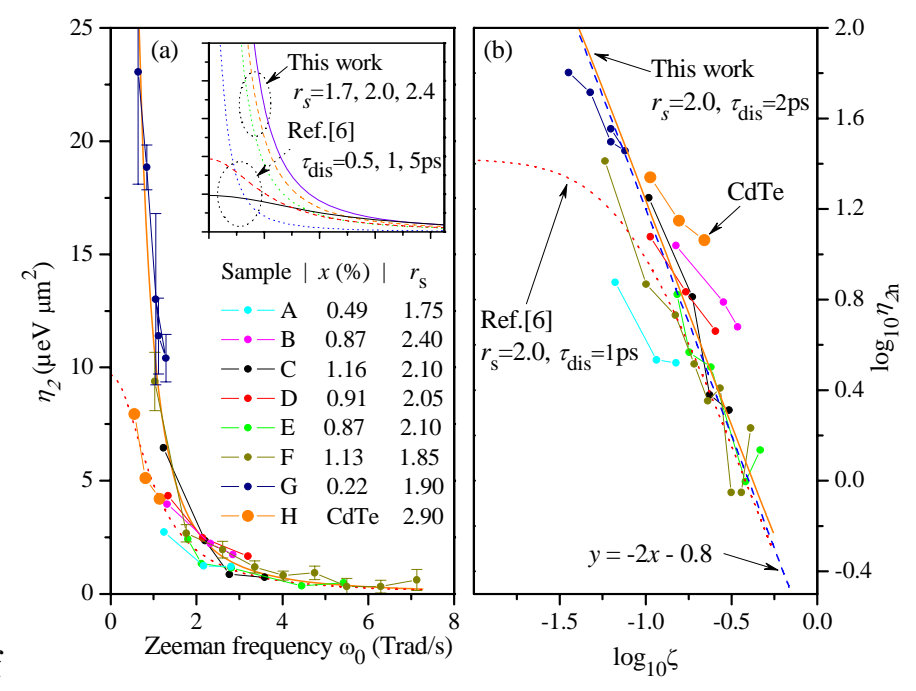

FIG. 3: (color online) $q^{2}$-damping and spin polarization. (a) $\eta_{2}$ is plotted as a function of $\omega_{0}$. Symbols are data obtained on samples with various $\mathrm{Mn}(x)$ and electron $\left(r_{s}\right)$ concentrations. Concentrations are measured as in Ref.[13]. Lines are calculated $\eta_{2}:$ using Eq.(7) with $r_{s}=2$ and $\tau_{d i s}=2 \mathrm{ps}$ (full line), using Ref. ${ }^{6}$ with $r_{s}=2$ and $\tau_{d i s}=1 \mathrm{ps}$ (dotted line). The insert shows variations of Eq.(7) with $r_{s}$ (upper curve is lower $r_{s}$ ) and variations of Ref. ${ }^{6}$ with $\tau_{\text {dis }}$ (flatter curve is lower $\tau_{\text {dis }}$ ). (b) Check of the power-law behavior : $\eta_{2 n}=\eta_{2} / \frac{\hbar^{2}}{2 m^{*}}$ is plotted as a function of the spin polarization $\zeta$ in a $\log _{10}$ frame. Lines corresponds to calculated $\eta_{2 n}$ from (a). All data curves are parallel to to $y=-2 x$.

that the observed phenomenom is not due to the presence of Mn impurities. CdTe data need additional treatment because of mass renormalization due to high magnetic fields. This is out of the scope. We will concentrate on the average behavior of CdMnTe data. First, the behavior for $\omega_{0} \rightarrow 0$ is impossible to explore below $1.0 \mathrm{Trad} / \mathrm{s}(0.65 \mathrm{meV})$ as such pulsations are beyond the rejection of the spectrometer. But in the range of explored pulsations, as shown in Fig.3(a) the average curve is very well reproduced by Eqs.(7)-(9) when taking a disorder scattering time $\tau_{d i s} \sim 2$ ps. For such a time scale, densities and experimental conditions, the spin-Coulomb drag coefficient $\frac{1}{\tau_{e e}}$ is always smaller than $15 \mathrm{~ns}^{-1}$ and has negligible impact on measurments. However, enhancement of both the Zeeman energy $\left(Z^{*} \geqslant Z\right)$ and the transverse field due to the Coulomb local exchange field are clearly needed to make the theory of Ref. ${ }^{6}$ match the experiment. Insert of Fig.3(a) shows how, even when varying the disorder time $\tau_{d i s}$, Eq. (20) of Ref. ${ }^{6}$ does not reproduce the data. These 
conclusions are clearer when plotting $\eta_{2 n}=\eta_{2} / \frac{\hbar^{2}}{2 m^{*}}$ as a function of the spin polarization $\zeta$ in a log-log frame as shown in Fig.3(b). Indeed, when $\omega_{0} \geqslant 1 \operatorname{Trad} / \mathrm{s}$ and for $r_{s} \sim 2$, the Zeeman enhancement $\omega_{0}^{*} / \omega_{0} \sim 2$, so $\left(\omega_{0}^{*} \tau_{d i s}\right)^{2} \gg 1$ and $\eta_{2 n}$ becomes :

$$
\lim _{\omega_{0}^{*} \tau_{d i s} \gg 1} \eta_{2 n} \simeq \frac{1}{\zeta^{2}} \frac{1}{E_{F}} \frac{\hbar}{\tau} 3\left[\frac{\omega_{0}^{*}}{\omega_{0}}-1\right]
$$

Comparing Eq.(10) and Fig.3(b), the $1 / \zeta^{2}$ behavior is fulfilled by the data even for the lowest measurable frequencies in agreement with $\eta_{2 n}$ calculated by Eq.(7). On the contrary, the $1 / \zeta^{2}$ behavior requests higher frequencies to appear in Eq.(20) of Ref. ${ }^{6}$. This confirms that spin-current dynamics is determined by individual pair dynamics for which the relevant precession pulsation is $\omega_{0}^{*}$ and not $\omega_{0}$. Moreover, the individual spin-flip scattering time has been probed by ERRS in the same conditions ${ }^{13}$ from $q=0$ spectra. At $q=0$, the energies of individual pairs are degenerate to $Z^{*}$ and presents a well defined peak in Raman spectra. Processing the linewidth of this peak with the same procedure gives a time in between $1.2 \mathrm{ps}$ and $1.8 \mathrm{ps}$ in very good agrement with the present determination of $\tau_{d i s}$.

In conclusion, we have demonstrated both experimental and theoretically that, in conducting systems, transverse propagating spin waves with momentum $q \neq 0$ carry a spincurrent, which despite its collective character is governed by single particle dynamics, where both disorder and spin-Coulomb drag play a role. Both mechanisms induce losses in the spin-current which damp the magnetization mode with a rate proportional to $q^{2}$. The form of this damping is intrisically linked to the kinetic motion of the spin carriers. Consequently, in the context of magnonics ${ }^{5}$ performed in $2 \mathrm{D}$ or $3 \mathrm{D}$ ferromagnetic metals, the propagation length $l_{\text {prop }}$ of a spin wave in a conducting system has to be optimized : assuming a group velocity $v_{g}=\beta q$ and a damping rate similar to Eq.(9), then $l_{\text {prop }}$ reaches its maximum $\beta \hbar / \sqrt{\eta_{0} \eta_{2}}$ for $q_{\max }=\sqrt{\eta_{0} / \eta_{2}}$.

The authors would like to thank M. Combescot and G. Vignale for fruitfull discussions as well as ANR grant GOSPININFO, German grant HA 5893/1-1 and Polish MNiSW grant N 202 054 32/1198.for financial supports.

1 A. Fert, S. Lee, Phys. Rev. B 53, 6554 (1996)

2 S. Murakami et al., Science 301, 1348 (2003) 
3 I. D'Amico and G. Vignale, Phys. Rev. B 62, 4853 (2000);E. M. Hankiewicz, G. Vignale, J. Phys.: Condens. Matter 21 (2009)

4 C. P. Weber et al., Letters to Nature 437, 1330 (2005)

5 D. S. Deng et al. , Phys. Rev. B 66, 104435 (2002);V. V. Kruglyak and R. J. Hicken, J. Magn. Magn. Mater. 306, 191 (2006)

6 E. M. Hankiewicz et al. Phys. Rev. B 78, 020404(R) (2008)

7 G. Karczewski at al., J. Cryst. Growth 184/185, 814 (1998)

8 B. Jusserand et al. Phys. Rev. Lett. 91, 086802 (2003)

9 F. Perez. Phys. Rev. B. 79, 045306 (2009)

10 J. A. Gaj et al., Solid State Comm. 29, 435 (1979)

11 G.Abstreiter, M.Cardona, and A .Pinczuk, Light Scattering in Solids IV, Springer (1984)

12 G. F. Giuliani and G. Vignale, "Quantum theory of the electron liquid", Cambridge university press $(2005)$

13 F. Perez et al., Phys. Rev. Lett. 99, 026403 (2007)

14 S. A. Crooker et al., Phys. Rev. Lett. 77, 2814 (1996) 